\title{
The prospects of on-demand urban air mobility in Zurich, Switzerland
}

\section{Working Paper}

\section{Author(s):}

Balać, Miloš; Rothfeld, Raoul L.; Hörl, Sebastian

Publication date:

2019

Permanent link:

https://doi.org/10.3929/ethz-b-000355676

Rights / license:

In Copyright - Non-Commercial Use Permitted

Originally published in:

Arbeitsberichte Verkehrs- und Raumplanung 1443 


\title{
The Prospects of on-demand Urban Air Mobility in Zurich, Switzerland
}

\author{
Milos Balac ${ }^{1}$, Raoul L. Rothfeld ${ }^{2}$ and Sebastian Hörl ${ }^{3}$
}

\begin{abstract}
Urban Air Mobility (UAM) is a novel concept based on the use of Vertical Takeoff and Landing vehicles for aerial transportation of people in urban settings. It can, potentially, disrupt the way people move today. The factors and impact of demand drivers for UAM, are, however, largely unknown. Therefore, the goal of this study is to present a methodology to investigate demand for UAM using an agentbased approach.

The methodology presented is used successfully to investigate UAM demand for a case study of Zurich, Switzerland, in showing demand sensitivity to various technological and operational parameters. This should provide a backbone for future research in other urban areas around the world and operational optimization of the UAM services.
\end{abstract}

\section{INTRODUCTION}

The advent of combustion engines and automobiles had substantial impact on life as we know it today. The automobile has brought freedom to travel to nearly every household in the developed world. This increase in accessibility has led to economic growth, prosperity, and an increase of wellbeing in general. Now, a century after Ford Motor Company first produced a car for the mass market, we are observing ever more negative effects that accompany the freedom, which the automobile has given us. Some of these evergrowing problems are congestion, parking space needs and fatalities. Congestion levels are soaring (i.e. in Mexico City people spend on average 59 minutes more on the roads during peak hours due to congestion [1]). Space devoted to parking is reaching staggering levels (at the moment, there exist more than three parking spaces per vehicle in the US [2]). And, the automobile was cause to 1.35 million fatalities in 2018 [3].

The negative side of the automobile has led researchers to promote more sustainable transportation modes, among them shared modes or future automated electric vehicle fleets. Recently, Uber Elevate has started to promote a new concept of on-demand mobility called Urban Air Mobility (UAM). The concept is based on airborne transportation of people in urban settings. While certain variations of this service already exist today (i.e. Voom [4], a subsidiary of AIRBUS in Sao Paulo, or BLADE, a service between Manhattan and NYC area airports [5]), they are all based on traditional helicopters. The UAM concept, on the other hand, relies

\footnotetext{
${ }^{1}$ Milos Balac is with the Institute for Transport Planning and Systems, ETH Zurich, Switzerland milos.balaceivt.baug.ethz.ch

${ }^{2}$ Raoul L. Rothfeld is with Technical University of Munich and Bauhaus Luftfart e.V., Taufkirchen, Germany raoul.rothfeld@bauhaus-luftfahrt. net

${ }^{3}$ Sebastian Hörl is with the Institute for Transport Planning and Systems, ETH Zurich, Switzerland sebastian.hoerleivt.baug.ethz.ch
}

mostly on electric Vertical Take-off and Landing (eVTOL) vehicles that promise to be more efficient and less noisy than traditional helicopters (see [6] on how some communities reacted to noise coming from BLADE helicopters). This way, they might increase acceptance of this kind of service by local communities and might provide a more energy-efficient and affordable type of transportation.

The concept promises to remove some traffic from the roads and to move it to the uncongested skies, thus reducing some of the negative effects of the car. While the positives of the concept are yet subject to research (some results can be found in [7], suggesting that under some circumstances VTOLs can be more energy efficient than ground vehicles), it is interesting to evaluate what may be the actual market penetration of such a service around the world. In the near future, as the technology is yet to mature, it is expected that the service will be costly and only accessible to the few, as was the case for both automobile and long-distance aircraft travel. In the long-term, as technology is progressing and economies of scale kick in, it is expected that the service could become widely affordable. Different stages of UAM integration in the transportation systems are well portrayed by [8] where it is stated that, eventually, UAM will scale economically to high-demand operations with minimal fixed costs.

In this paper, we investigate the potential market for an on-demand UAM service for a city like Zurich, Switzerland. While the city itself is small in size, its metropolitan region can be suitable for the implementation of such a service.

\section{BACKGROUND}

So far, researchers have mainly been focusing on the technical side of eVTOL vehicles and their integration in the air space. However, there is some research on integration of the UAM concept into the transportation system and its potential demand. [9] presents plans for an extensive Stated Preference (SP) survey of high-income commuters in Atlanta, Boston, Dallas-Ft. Worth, San Francisco and Los Angeles to understand potential customers' willingness to pay for on-demand flights in urban areas. The main target group of the planned survey are commuters with commuting time longer than $45 \mathrm{~min}$. [10] describes an SP survey in Munich, Germany, to capture people's preference towards UAM as a transportation mode. They find that travel time, travel cost, and safety may be critical determinants for the adoption of UAM.

[11] shows how the demand for a UAM service might look like in Zurich, Switzerland, by allowing for pick-up and drop-off to take place anywhere in the study area. The study 
shows that vehicle speed and access time have substantial impact on demand. Moreover, the results show that cost structures determine the customer segment for which a UAM fleet is attractive.

[12], [13] further documents the development of a framework for the estimation of potential demand of UAM concepts. This paper builds on the framework to perform an extended case-study for a UAM service in Zurich, taking into account added functionality such as intermodality, vertiport placement, and aerial network design.

\section{Methodology}

\section{A. Simulation environment}

The UAM framework is set up using eqasim ${ }^{1}$, a novel extension to the widely-known agent-based transport simulation framework MATSim [14]. The MATSim framework provides a queue-based network simulation layer, in which movements of large numbers of agents can be simulated at the same time. From competition over limited capacity in the network traffic, jams and delays emerge in the system, which can be analyzed at road-level and per-second detail. The resulting travel characteristics, such as wait time, travel time, and cost, are then used as a basis to modify agents' daily plans. Those consist of multiple activities throughout one day and their connecting trips. By running network simulation and a behavioral decision making component iteratively, the system converges to a stable state in which all agents' plans reside in stochastic user equilibrium, i.e. all agents have adapted their plans to the plans of all others and have reached their local optimum. Unlike the more general MATSim, where an evolutionary algorithm is applied to simulate decision-making for transport modes, departure times or even activity locations, eqasim uses discrete choice models to specifically investigate choice of transport mode for the travelers. It thereby aims at connecting agent-based transport simulation with traditional discrete choice theory and its statistical models which can be directly estimated from survey data.

The choice models are applied in the following way: before each network simulation, a share of agents (usually around 20\%) is allowed to change the mode of transport for all of their daily trips. From the perspective of mode choice, these trips can be seen as a sequence of transportation modes. As a first step, the framework constructs the full set of mode sequences with which an agent's daily plan could be covered, considering trip- and tour-level constraints, as well as the availability of certain modes of transport to the agent. For instance, an agent without a driving license would not be allowed to use "car" as a transport mode and in any case a car can only be used for a trip if it is driven to its origin location before. Each sequence is then assigned a utility value based on travel time, wait time, cost, etc. for the selected modes. The framework then allows for various choice processes on these utilities, the most widely known being a Multinomial Logit selection. For an in-depth discussion of the choice

${ }^{1}$ http://www.eqasim.org process, constraints and relation to the standard MATSim approach, we'd like to refer the reader to [15] and [16]. After decisions for all agents are made, the network simulation is performed again. Measured travel characteristics such as travel time, wait time and others are then used in the next round of decision making as explained above.

As a basis for the integration of UAM, developments by [12] are utilized and extended which provide UAM as an additional mode of transport within eqasim. For this, definitions of UAM vertiports, flight routes, and VTOL vehicles are provided which include, among other information, the location of vertiports, their accessibility with ground-based transport modes, the take-off, landing, and cruising speeds of UAM vehicles, and their passenger capacity.

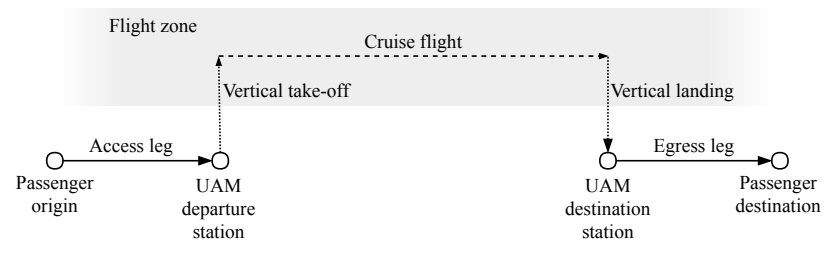

Fig. 1. Illustration of an exemplary UAM trip

Each UAM trip consists of three legs: (1) access, (2) flight, and (3) egress leg; with the flight leg itself being split into three states: vertical take-off, cruise flight, and vertical landing, as illustrated in Figure 1. A router component is responsible to provide a combination of access, flight, and egress legs that generates the highest utility for the agent. Available access and egress modes can be defined in advance. In this study, they are walk, bike, public transport, taxi, and a car-sharing service. The search for the best starting and ending UAM station also takes into account the potential wait time at each of the UAM stations. The router feeds the complete route and total utility of the UAM trip to the modechoice model which then chooses the sequence of modes the agent will use in its daily plan as described previously.

During the simulation, a passenger request is created once an agent departs on a UAM access leg. These requests are collected in a request queue, and handled on a first-in first-out basis. Upon arriving at the departure UAM station, the passenger has to traverse boarding processes which are defined via pre- and post-flight process times. In case the requested UAM vehicle has not been present at the departure station or did not arrive before the passenger did, the passenger has to wait for the requested vehicle before starting the preflight boarding process. Once the vehicle and the passenger have completed the pre-flight process, they take-off vertically until the pre-defined flight level is reached at which point the vehicle follows the shortest flight path to the destination UAM station at cruising speed. Once the destination station has been reached, following a VTOL descent, the vehicle and its passenger have to complete post-flight processes which also take a pre-defined amount of time. Lastly, the passenger departs from the destination station on an egress leg. The passenger's trip is completed upon arrival at the passenger's destination. 
On the vehicle side, the dispatching algorithm works as follows: at any point in time the dispatcher knows about all pending requests in the system. As soon as a request emerges at a vertiport an idle vehicle at that station is assigned to the request to await the passenger. If no vehicle is available at the vertiport, an idle vehicle at another location is sent immediately on a empty flight towards that station and the request is assigned to that vehicle. In case of high demand, where no idle vehicles are available, the vehicles are dispatched to vertiports with waiting passengers, following the order in which the requests have arrived. However, in the experiments presented here, an unlimited number of vehicles is used which means that the dispatcher always has a vehicle available at the origin landing station.

\section{SCENARIO}

\section{A. Travel demand}

The eqasim scenario for Zurich is based on a couple of data sets, which are open to research within Switzerland. First, sociodemographic attributes are derived from a detailed census data set. Using this information, households consisting of around 8 million synthetic persons with attributes such as age, gender, and car availability are generated. Additionally, home and work locations are assigned to the people such that the sociodemographic structure resembles reality from a spatial perspective. Afterwards, the countrywide HTS (household travel survey) [17] is used to attach daily activity chains to the synthetic population by statistical matching. This way, each of the synthetic persons receives a sequence of trips that need to be performed on an average week day. Finally, a subset of the population is obtained only including agents which interact with an area of $30 \mathrm{~km}$ around Zurich. To arrive at acceptable simulation times, a $10 \%$ random sample of those agents is created. The travel demand used in this study therefore consists of around 160000 agents with around 580000 trips that need to be simulated.

Travelers using Zurich Airport are modeled separately as they are not included in the standard Zurich scenario. We distinguish between Swiss residents and visitors and also between economy, business, and first class travelers. The summary of the number of passengers belonging to each of these groups can be seen in Table I, which is based on two publications by Zurich Airport [18], [19]. Information on origins and destinations within Switzerland is not available. As an approximation we distribute travelers based on population densities within the study area.

TABLE I

AIRPORT PASSENGERS AND THEIR COST ELASTICITIES

\begin{tabular}{l|rrr}
\hline & Locals & Visitors & $\lambda_{\text {income/cost }}$ \\
\hline Economy Class & 17,064 & 15,133 & -0.5 \\
Business Class & 1,618 & 1,435 & -0.8 \\
First Class & 59 & 52 & -0.95 \\
\hline
\end{tabular}

\section{B. Mode choice model}

The mode choice model used in this study is a multinomial logit model based on a stated preference study [20] for the canton of Zurich. The corresponding utility functions are given in Equations 1 through 4 while the model parameters are shown in Table II. Per-trip choice dimensions are denoted as $x$, per-agent attributes are denoted as $a$, model constants as $\theta$ and estimated model parameters are given as $\alpha$ for the alternative-specific constants and $\beta$ for marginal utility parameters. Travel costs for the specific trips are denoted as $c$ and calculated based on distance for the conventional modes.

As the survey does not take into account taxi ${ }^{2}$ or UAM, the parameters of those alternatives (Equations 5 and 6) are assumed to be the same as for public transport. Please refer to the discussion part of this paper for possible implications of that assumption.

To produce the correct mode shares in total and per distance class in eqasim, some parameters need to be adjusted: $\alpha_{\text {walk }}$ is adjusted to $1.3, \alpha_{\text {bike }}$ is adjusted to 0.4 , and $\beta_{\text {travelTime,bike }}$ is changed to -0.09 . The largest change takes place for the alternative specific constant of walking. This is necessary to compensate for the under-sampling of very small distances in the survey data.

Furthermore, components are added to the model to account for additional features of the UAM scenario. An income elasticity is added to the model to better incorporate people's sensitivity to cost into the model. A value of $\lambda_{\text {income/cost }}=-0.15$ is used as has been found for Switzerland in prior research [21]. Also based on [21], different elasticities are assumed for travelers from Zurich airport such that they represent the profiles of economy, business, and first class travelers (see Table I).

$$
\begin{aligned}
u_{\text {walk }}= & \alpha_{\text {walk }}+\beta_{\text {travelTime,walk }} \cdot x_{\text {travelTime,walk }} \\
u_{\text {bike }}= & \alpha_{\text {bike }}+\beta_{\text {travelTime,bike }} \cdot x_{\text {travelTime,bike }} \\
& +\beta_{\text {age,bike }} \cdot \max \left(a_{\text {age }}, 18\right) \\
u_{\text {car }}= & \alpha_{\text {car }}+\beta_{\text {travelTime,car }} \cdot x_{\text {travelTime,car }} \\
& +\beta_{\text {cost }} \cdot\left(\frac{x_{\text {crowflyDistance }}}{\theta_{\text {averageCrowflyDistance }}}\right)^{\lambda_{\text {distance/cost }}} \\
& \cdot\left(\frac{a_{\text {income }}}{\theta_{\text {averageIncome }}}\right)^{\lambda_{\text {income/cost }}} \cdot c_{c a r} \\
u_{\mathrm{pt}} & \alpha_{\mathrm{pt}}+\beta_{\text {travelTime,pt }} \cdot x_{\text {travelTime,pt }} \\
& +\beta_{\text {accessEgressTime,pt }} \cdot x_{\text {accessEgressTime,pt }} \\
& +\beta_{\text {waitTime,pt }} \cdot x_{\text {waitTime,pt }} \\
& +\beta_{\text {transfer,pt }} \cdot x_{\text {transfers,pt }} \\
& +\beta_{\text {cost }} \cdot\left(\frac{x_{\text {crowflyDistance }}}{\theta_{\text {averageCrowflyDistance }}}\right)^{\lambda_{\text {distance/cost }}} \\
& \cdot\left(\frac{a_{\text {income }}}{\theta_{\text {averageIncome }}}\right)^{\lambda_{\text {income/cost }}} \cdot c_{\mathrm{pt}}
\end{aligned}
$$




$$
\begin{aligned}
u_{\text {taxi }}= & \alpha_{\text {taxi }}+\beta_{\text {travelTime,taxi }} \cdot x_{\text {travelTime,taxi }} \\
& +\beta_{\text {waitTime,taxi }} \cdot x_{\text {waitTime,taxi }} \\
& +\beta_{\text {cost }} \cdot\left(\frac{x_{\text {crowflyDistance }}}{\theta_{\text {averageCrowflyDistance }}}\right)^{\lambda_{\text {distance/cost }}} \\
& \cdot\left(\frac{a_{\text {income }}}{\theta_{\text {averageIncome }}}\right)^{\lambda_{\text {income/cost }}} \cdot c_{\text {taxi }}
\end{aligned}
$$$$
u_{\mathrm{UAM}}=\alpha_{\text {uam }}+\beta_{\text {travelTime, } \mathrm{UAM}} \cdot x_{\text {travelTime,UAM }}
$$$$
+\beta_{\text {cost }} \cdot\left(\frac{x_{\text {crowflyDistance }}}{\theta_{\text {averageCrowflyDistance }}}\right)^{\lambda_{\text {distance/cost }}}
$$$$
\cdot\left(\frac{a_{\text {income }}}{\theta_{\text {averageIncome }}}\right)^{\lambda_{\text {income/cost }}} \cdot c_{\mathrm{UAM}}
$$$$
+\beta_{\text {transfer,UAM }} \cdot x_{\text {transfers,UAM }}
$$$$
+u_{\text {accessMode }}+u_{\text {egressMode }}
$$

TABLE II

MUlTiNOMIAL LOGIT MODEL USED TO PERFORM MODE DECISIONS IN THE eqasim SIMULATION [20]

\begin{tabular}{l|rrl}
\hline Mode & Variable & Parameter & \\
\hline Walking & $\alpha_{\text {walk }}$ & 0.631 & \\
& $\beta_{\text {travelTime,walk }}$ & -0.141 & $\mathrm{~min}^{-1}$ \\
\hline Cycling & $\alpha_{\text {bike }}$ & 0.344 & \\
& $\beta_{\text {travelTime,bike }}$ & -0.080 & $\mathrm{~min}^{-1}$ \\
& $\beta_{\text {age,bike }}$ & -0.049 & $\mathrm{a}^{-1}$ \\
\hline Car & $\alpha_{\text {car }}$ & 0.827 & \\
& $\beta_{\text {travelTime,car }}$ & -0.067 & $\mathrm{~min}^{-1}$ \\
\hline Public Transport & $\alpha_{\mathrm{pt}}$ & 0.000 & \\
& $\beta_{\text {travelTime,pt }}$ & -0.019 & $\mathrm{~min}^{-1}$ \\
& $\beta_{\text {accessEgressTime,pt }}$ & -0.080 & $\mathrm{~min}^{-1}$ \\
& $\beta_{\text {waitTime,pt }}$ & -0.038 & $\mathrm{~min}^{-1}$ \\
& $\beta_{\text {transfer,pt }}$ & -0.170 & 1 \\
\hline Cost & $\beta_{\text {cost }}$ & -0.126 & $\mathrm{CHF}$ \\
& $\lambda_{\text {distance/cost }}$ & -0.400 & \\
\hline Constants & $\theta_{\text {averageCrowflyDistance }}$ & 40 & $\mathrm{~km}$ \\
& $\theta_{\text {averageIncome }}$ & 9,000 & $\mathrm{CHF}$ \\
\hline
\end{tabular}

\section{UAM service definition}

UAM is added to the Zurich scenario with predefined locations for UAM vertiports, as illustrated in Figure 2. These stations are selected based on local expertise of commuting flows within the greater Zurich area.

All stations are assumed to be directly connected to every other station by beeline flight paths, including a $+20 \%$ detour factor to realistically mimic deviations from the optimal path during flight. This is expected to occur in actual UAM operation. Further, VTOL duration, i.e. the time required to traverse the vertical distance from UAM vertiports to UAM flight paths $(600 \mathrm{~m}$ above ground), is assumed to be $60 \mathrm{~s}$. Fleet sizing and landing station capacity are out of this study's scope. Thus, while conceptually part of the framework, fleet size, and stations capacities were considered unlimited.

Since the Zurich scenario is run using a $10 \%$ population sample, passenger capacity for UAM vehicles would need to be scaled down, respectively. Scaling down passenger capacities of UAM vehicles ten-fold poses substantial issues since the majority of next-generation VTOL projects are expected to provide capacity for less than six passengers [22]. Thus, UAM passenger pooling, i.e. ride-sharing, is disabled in the scope of this study and will be examined in future research. For now, unit capacity is assumed for every vehicle to simplify interpretation of simulation results.

\section{EXPERIMENTS}

\section{A. Setup}

This study aims at understanding demand potentials for UAM based on different VTOL vehicle cruising speeds, processing times at take-off and landing stations, and pricing structure. These values, along with the accessibility of vertiports, which is also taken into account here, are crucial for the success of UAM operations. However, neither current literature nor technological developments provide a clear picture of these parameters, and therefore a wide range of values is tested.

The set of values that are used in the experiments can be seen in Table III. While in the simulation take-off and landing times are treated separately, agents react the same to both so we are only interested in the total process time which is shown in Table III. Process times in the experiments range from 0 to $12 \mathrm{~min}$. These times include security check, boarding, and other necessary procedures at the UAM vertiports. Zero process time is included in the experiments to reflect a potentially seamless boarding procedure as is the case for public transport services. Cruising speed of vehicles is tested for 60,120 , and $240 \mathrm{~km} / \mathrm{h}$ to cover a broader range of vehicles that might be used in practice. While there are some indications on possible process times and vehicle speeds based on current technological developments and experiences from VOOM and BLADE operations, costs structure that would make the service profitable for the operator is difficult to predict. We, therefore, initially rely on Uber's estimate that in the long-run fees would be similar to those of Uber X. A base fare of $6 \mathrm{CHF}^{3}$ is set along with varying variable cost ranging from 0.6 to $4.2 \mathrm{CHF} / \mathrm{km}$. Reducing the fare under $0.6 \mathrm{CHF} / \mathrm{km}$ seams unreasonable as that value is below the current cost of car ownership.

TABLE III

EXPERIMENTS SETUP

\begin{tabular}{l|r}
\hline & Values Tested \\
\hline Process Times [min] & $0,4,8,12$ \\
Cruising Speed [km/h] & $60,120,240$ \\
Variable Cost [CHF/km] & $0.6,1.2,1.8,2.4,3.0,3.6,4.2$ \\
\hline
\end{tabular}

\section{B. Results}

Figures 2 and 4 show the flow of passengers between vertiports for scenarios with a cruising speed of $240 \mathrm{~km} / \mathrm{h}$ and no process time, and variable cost of $0.6 \mathrm{CHF} / \mathrm{km}$ and 1.2 $\mathrm{CHF} / \mathrm{km}$ respectively. Passenger flows in Figure 2 show

${ }^{3} 1 \mathrm{CHF}=1.00$ USD $(1$ April 2019, xe.com $) ; 1 \mathrm{CHF}=0.73$ USD based on purchasing power parity (March 2019, [23]) 
that the majority of UAM trips are from/to Winthertur and Zug, which are the stations furthest from the center of Zurich (located between Zurich HB and Bellevue stations). Also the connection between Baden and ETH Hönggerberg (large university campus) has strong flow, which is understandable as public transport connection between these two locations involves several transfers. Stations at the Lake of Zurich also have larger than the average flows, mainly because the lake is a natural obstacle and both public transport and car travel involves long detours. With increasing variable cost some of the long-distance UAM flows disappear as can be seen in Figure 4.

Experiments with variable costs exceeding $1.8 \mathrm{CHF} / \mathrm{km}$ show that a UAM service could attract very few customers for these pricing structures, which makes the service only attractive for the very high-income segment of the population. As this is not the scope of this paper, the results are focused only on the experiments with variable costs of up to $1.8 \mathrm{CHF} / \mathrm{km}$.

Figures 5, and 6 show the number trips, and mean beeline distance for the UAM service for different scenario setups.

It is clear that both processing time and cruising speed have a non-marginal effect on the total number of trips.

Looking at Figure 5 one can see that an increase in cruising speed from $60 \mathrm{~km} / \mathrm{h}$ to $120 \mathrm{~km} / \mathrm{h}$ has a stronger effect on the number of trips, than an increase from $120 \mathrm{~km} / \mathrm{h}$ to $240 \mathrm{~km} / \mathrm{h}$. This is especially important when looking at the trade-off between cruising speed and process time. It is noticeable that doubling the cruising speed can compensate for additional $4 \mathrm{~min}$ of process time, but this effect is diminishing as with higher speeds the marginal reduction of travel time is smaller.

Increasing per distance costs reduces average beeline travel distance for UAM trips (Figure 6). As the speed of VTOL vehicles increases, passengers start to take UAM for longer trips, as the reduction in travel time compensates the costs. Increases in the per distance cost reduce the average UAM trip distance as longer trips are no longer able to compensate additional cost with travel time reductions. This also supports findings in Figures 2 and 4. Process time does not seem to have a clear direction of influence. One noticeable irregularity is for experiments with $1.2 \mathrm{CHF} / \mathrm{km}$ and $1.8 \mathrm{CHF} / \mathrm{km}$ variable cost, and $60 \mathrm{~km} / \mathrm{h}$ cruising speed and 0 processing time, where an increase in the average UAM trip distance appears. This effect can only be explained as a consequence of using a sample population and having very low demand for these cases.

Figure 3 shows that most of the departures for UAM trips take place during the morning and afternoon peak hours and that the usual midday peak, which exists for car trips, is missing. As the roads are most congested during morning and evening rush hours, those are the times where UAM can substantially reduce travel time for its users. The evening peak is wider since at those times the number of activities is higher. Also, they are spread over longer period of time as people do shopping and leisure activities after work hours.

So far, the results presented are based on the base fare of

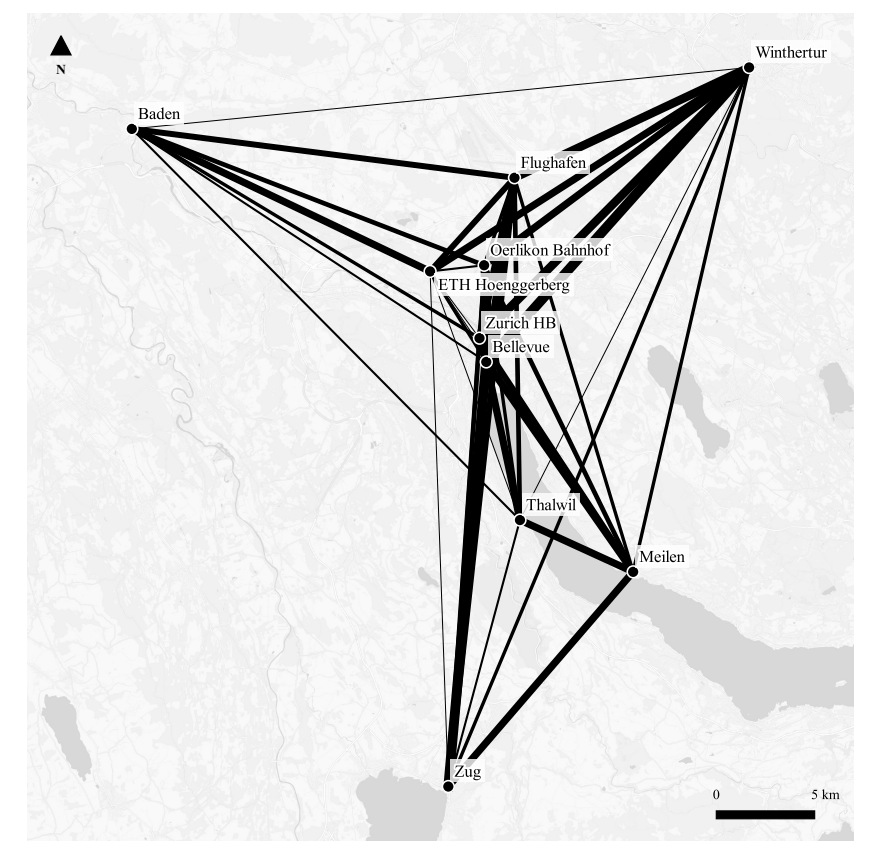

Fig. 2. Zurich UAM network with number of flights indicated by line thickness for variable cost of $0.6 \mathrm{CHF} / \mathrm{km}$, cruise speed of $240 \mathrm{~km} / \mathrm{h}$, and no process time.

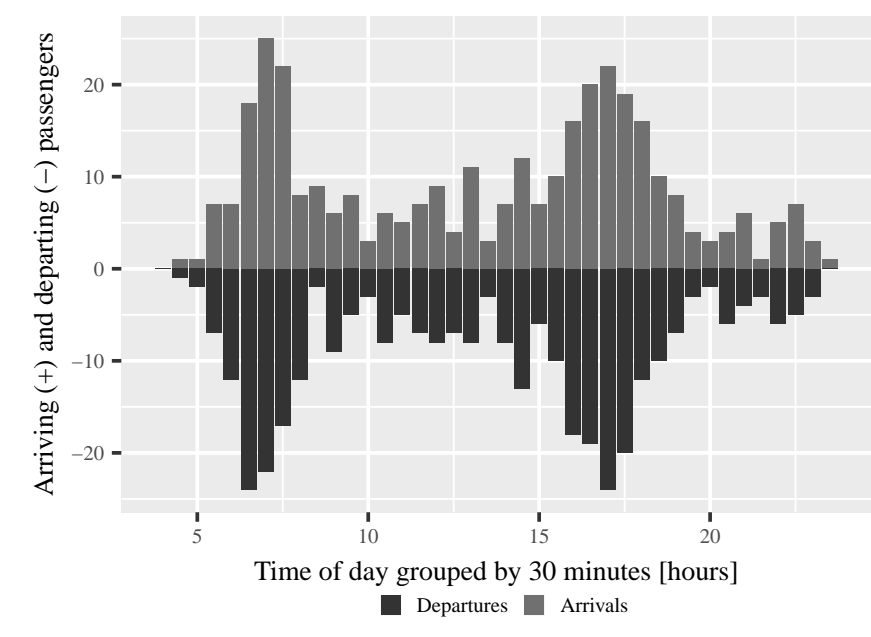

Fig. 3. Arrival and departure times of UAM passengers for the $0.5 \mathrm{CHF} / \mathrm{km}$, $240 \mathrm{~km} / \mathrm{h}$ and no process time experiment

6 CHF for a UAM trip. This value might be too optimistic as the overhead cost of operating a VTOL vehicle fleet together with landing fees might force the operator to charge a higher base fare for each UAM flight in the initial phase of operations. Figure 7 shows the influence of various base fares on the total amount of trips when the distance price is $0.6 \mathrm{CHF} / \mathrm{km}$, process time is $4 \mathrm{~min}$ and cruising speed is $240 \mathrm{~km} / \mathrm{h}$. Doubling the initial base fare to $12 \mathrm{CHF}$ almost reduces the demand threefold. It is also noticeable that the marginal decrease of demand does not linearly follow the increase in base fare, showing that some passengers are not as sensitive to price as others. Trips to and from the airport start impacting the demand for base fares below $12 \mathrm{CHF} / \mathrm{km}$. 
As the Zurich airport is well connected by public transport services and taxi fares are otherwise cheaper than UAM this is expected.

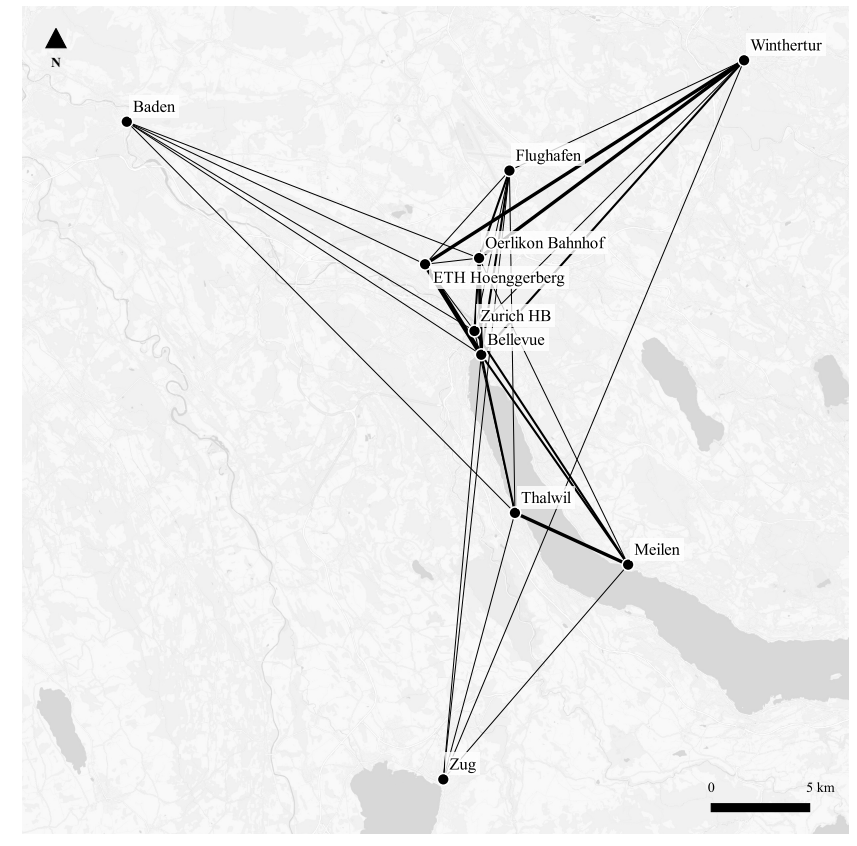

Fig. 4. Zurich UAM network with number of flights indicated by line thickness for variable cost of $1.2 \mathrm{CHF} / \mathrm{km}$, cruise speed of $240 \mathrm{~km} / \mathrm{h}$, and no process time

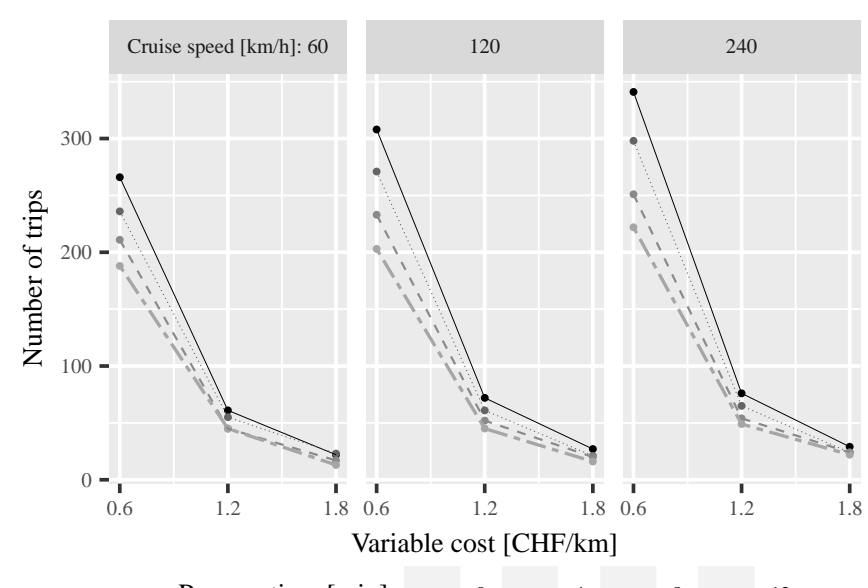

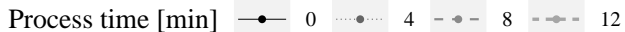

Fig. 5. Number of trips based on scenarios with varying variable cost, cruise speed, and process times

\section{DISCUSSION}

Comparing the results to earlier work on estimating potential demand for UAM in Zurich [11], it is noticeable that the estimated demand is drastically lower when process times, access/egress trips, and infrastructure placement are included in the decision process. While the present work does not cover all possible combinations of speed, cost, and process time it still presents some important results: faster VTOL vehicles might not be able to compensate

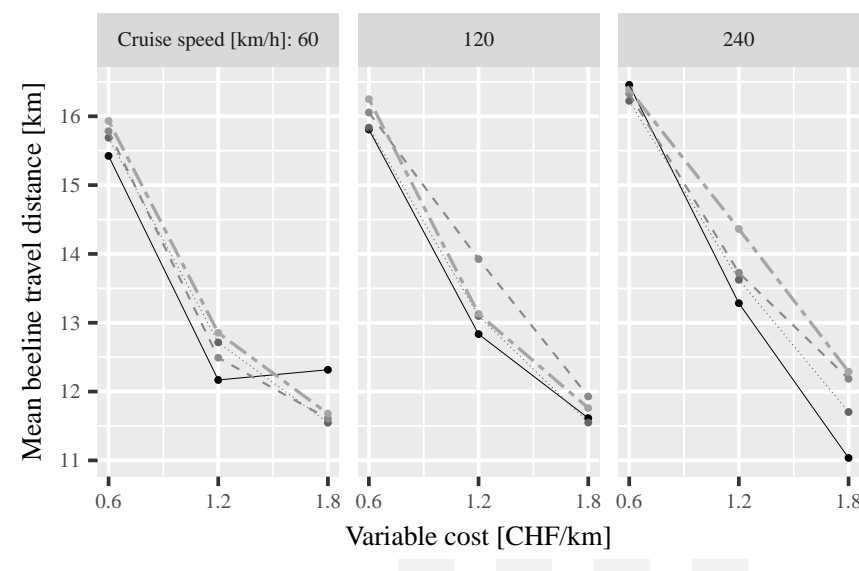

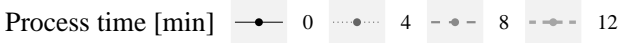

Fig. 6. Mean travel beeline distance $[\mathrm{km}]$ based on scenarios with varying variable cost, cruise speed, and process times

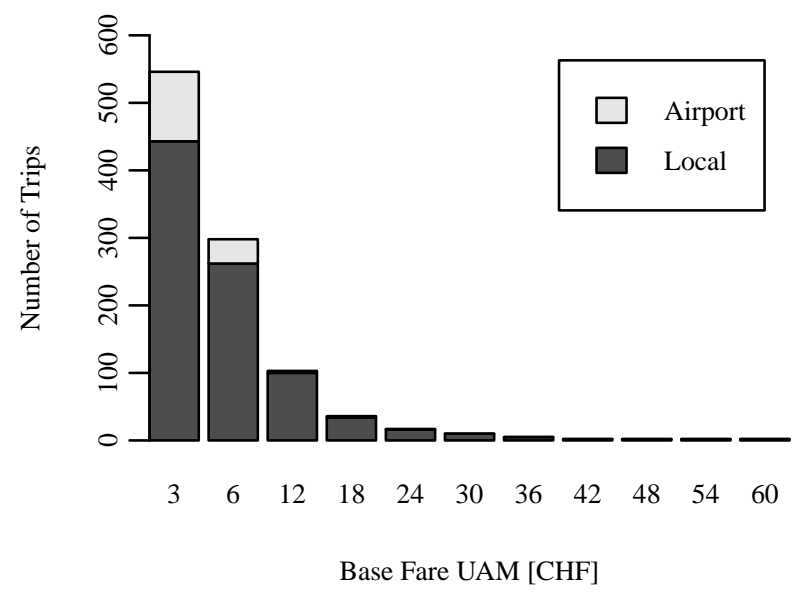

Fig. 7. Demand for UAM based on different base fare for local and airport trips

for additional process time, even though they can attract more users. Moreover, as faster VTOL vehicles might have a higher marginal cost of operation, they might be less profitable. Doubling the base fare has a smaller negative influence on the demand than doubling variable costs, which is interesting from the operator perspective, and comes as a consequence of mean trip distances served.

Unlike car and public transport, the UAM concept would not have a large impact on the urban design as the only infrastructure that is required are landing stations. As VTOL technology is maturing, high noise levels that are a problem associated with helicopters may no longer be an issue. This could attract public transport providers to include this kind of service in their operations, which would allow for reduced overhead and landing fees, therefore increasing the demand for the service. 


\section{A. Limitations and Outlook}

The simulated population is based on a $10 \%$ sample. While this allows for shorter computation times and enables to test a multitude of scenarios (94 were simulated here), it introduces certain statistical error. This is especially true for cases where the number of UAM trips is very low $(<10)$. However, the results provide a good indication of the general demand for UAM, based on different vehicle and infrastructure characteristics. It is, nevertheless necessary to simulate a $100 \%$ sample scenario when the goal is optimization of fleet control, perhaps even including pooling of similar trip requests. The methodology used in this paper is ready for such problems and they will be tackled in future work.

In this work, passenger capacity of VTOL vehicles is constrained to one. While this prevents pooling of passengers it should not be considered as a limiting factor of this study as the number of UAM trips is rather low, making pooling inconvenient. However, in order to reduce per passenger costs, pooling will probably be necessary. This way operators of UAM will be able to attract larger demand. Therefore, it will be important to investigate how pooling impacts the demand, where it is feasible and how it affects the per passenger costs, and ultimately demand.

This paper investigates on-demand UAM services. The potentials of a scheduled UAM service is therefore not investigated. As scheduled flights might operate at lower cost by pooling passengers, its operation might be financially better for both operators and passengers. This kind of service might be more attractive to the operators in the initial phase of UAM passenger service. Therefore, its optimization and planning is a viable future research topic.

The number of available vehicles in the simulated experiments is unconstrained in order to find the upper bound for demand under different scenario setups. As this is practically infeasible, future work will need to investigate how different fleet sizes, dispatching, landing station sizing and flight scheduling can be optimized to both user and operator satisfaction. It is worth noting that the methodology presented here is ready to deal with those questions, but they are out of scope for this paper.

This work does not make assumptions on the level of automation of VTOL vehicles. In the starting phase of UAM operations these vehicles would certainly be piloted, and in the later stage would transition to fully automated. This change in automation will definitely impact the passenger fees but, the extent is unknown. While the exact fee structure for UAM service is hard to predict given the novelty of the service, the broad range of fee structures tested here provide valuable information on responsiveness of the demand to costs.

Automated vehicles (AVs) are not used as a possible competition to UAM in this study. While simulating AVs is certainly possible within the eqasim framework it was omitted here in order to avoid additional assumptions on AV market characteristics which would limit the findings presented here.
The mode-choice parameters for UAM used in this study are not estimated based on empirical data as they do not exist for Zurich. Since UAM can be considered more similar to public transport services than to private cars, approximation with public transport parameters seems reasonable. However, conducting stated preference surveys will be important to further refine these assumptions and results.

\section{CONCLUSION}

This paper presents one of the first detailed analyses of the potential demand for a UAM service. In spite of some limitations this paper makes several important contributions:

- versatile extension of eqasim able to simulate future UAM services is developed and presented

- the extension is able to evaluate and simulate intermodal UAM trips thus allowing for better comparison of travel alternatives

- sensitivity analysis of both marginal and base costs of UAM is presented.

The study is centered in Zurich, which is characterized by small population size, low population density, good public transport service and a high average income population. Our findings show that the demand for a UAM service for an urban area such as Zurich is rather small. However, the results presented show some important trade-offs between vehicle characteristics, price structure, and processing times at take-off and landing stations. Therefore, the methodology and findings presented here can drive the focus of future work and UAM implementation endeavors.

While these results suggest that UAM might only have a small share of the transportation market in a city like Zurich, this might not be the case for larger cities, with highpopulation density that are heavily congested, and characterized by poor public transport service levels. Future research centered on other cities is needed to obtain a complete picture of the full potential of UAM for transportation of passengers. The methodology presented in this paper is meant to facilitate further studies in different areas around the world to foster the understanding of the potential demand for Urban Air Mobility.

\section{REFERENCES}

[1] TomTom, "Congestion in Mexico City," 2019. [Online]. Available: www.tomtom.com/

[2] C. McCahill and N. Garrick, "Parking supply and urban impacts," in Parking Issues and Policies, S. Ison and C. Mulley, Eds. Emerald Group Publishing Limited, 2014, pp. 33-55.

[3] WHO, "Global status report on road safety 2018," Geneva, Tech. Rep., 2018.

[4] VOOM, "VOOM: an AIRBUS Company," 2019. [Online]. Available: https://www.voom.flights/en

[5] BLADE, "BLADE flights," 2019. [Online]. Available: https://blade.flyblade.com/

[6] Barron, James, "The Citizens of East Hampton v. Its Airport," 2017. [Online]. Available: https://www.nytimes.com/2017/07/04/nyregion/east-hamptonairport-noise-regulations.html

[7] A. Kasliwal, N. J. Furbush, J. H. Gawron, J. R. McBride, T. J. Wallington, R. D. De Kleine, H. C. Kim, and G. A. Keoleian, "Role of flying cars in sustainable mobility," Nature communications, vol. 10, no. 1555,2019 
[8] D. P. Thipphavong, R. Apaza, B. Barmore, V. Battiste, B. Burian, Q. Dao, M. Feary, S. Go, K. H. Goodrich, J. Homola, et al., "Urban air mobility airspace integration concepts and considerations," in 2018 Aviation Technology, Integration, and Operations Conference, 2018.

[9] L. A. Garrow, M. Ilbeigi, and Z. Chen, "Forecasting demand for on demand mobility," in 17th AIAA Aviation Technology, Integration, and Operations Conference, 2017.

[10] M. Fu, R. Rothfeld, and C. Antoniou, "Exploring preferences for transportation modes in an urban air mobility environment: a munich case study," in 98th Annual Meeting of the Transportation Research Board, TRB, Ed. Washington, D.C.: Transportation Research Board, January 2019.

[11] M. Balac, A. R. Vetrella, R. Rothfeld, and B. Schmid, "Demand estimation for aerial vehicles in urban settings," 2018, paper accepted for publication in IEEE Intelligent Transportation Systems Magazine.

[12] R. Rothfeld, M. Balac, K. Ploetner, and C. Antoniou, "Agent-based Simulation of Urban Air Mobility," in 2018 Modeling and Simulation Technologies Conference, AIAA, Ed. Atlanta, Georgia: AIAA AVIATION Forum, June 2018.

[13] R. Rothfeld, M. Balac, K. O. Ploetner, and C. Antoniou, "Initial analysis of urban air mobilitys transport performance in sioux falls," in 2018 Aviation Technology, Integration, and Operations Conference, AIAA, Ed. Atlanta, Georgia: AIAA AVIATION Forum, June 2018.

[14] A. Horni, K. Nagel, and K. W. Axhausen, The Multi-Agent Transport Simulation MATSim. London: Ubiquity Press, 2016.

[15] S. Hörl, M. Balac, and K. W. Axhausen, "A first look at bridging discrete choice modeling and agent-based microsimulation in matsim," Procedia Computer Science, vol. 130, pp. 900 - 907, 2018.

[16] _ "Pairing discrete mode choice models and agent-based transport simulation with MATSim," 98th Annual Meeting of the Transportation Research Board, 2019.

[17] Federal Statistical Office, Federal Office for Spatial Development, "Mobility in switzerland: Results from the microcensus mobility and transport 2015," 2017.

[18] F. Platten, F. Attinger, and L. Mischke, "Passenger flows," Flughafen Zrich AG, Zurich, Tech. Rep., 2018.

[19] Z. Airport, "Facts and figures 2017," Flughafen Zrich AG, Zurich, Tech. Rep., 2017.

[20] S. Hörl, F. Becker, T. Dubernet, and K. W. Axhausen, "Induzierter Verkehr durch autonome Fahrzeuge: Eine Abschätzung," Tech. Rep., Ongoing project SVI 2016/001.

[21] K. W. Axhausen, S. Hess, A. König, G. Abay, J. J. Bates, and M. Bierlaire, "Income and distance elasticities of values of travel time savings: New swiss results," Transport Policy, vol. 15, no. 3, pp. 173$185,2008$.

[22] M. Shamiyeh, J. Bijewitz, and M. Hornung, "A Review of Recent Personal Air Vehicle Concepts," in Aerospace 6th CEAS Conference. Bucharest: Council of European Aerospace Societies, October 2017.

[23] OECD, "Purchasing power parities (PPP), Monthly comparative price levels," 2019. [Online]. Available: https://stats.oecd.org/Index.aspx?DataSetCode=CPL 